\title{
Validación de ecuaciones antropométricas para evaluar composición corporal en niños preescolares chilenos
}

\author{
Marcia Velásquez $\mathbf{R}^{1 \mathbf{a}}$, Gabriela Salazar $\mathbf{R}^{\mathbf{1 b}}$, \\ Fernando Vio del $\mathbf{R}^{1}$, Nora Díaz $\mathbf{Z}^{1 \mathrm{lc}}$, Alyerina Anziani $\mathbf{G}^{1 \mathrm{ld}}$. \\ Validation of equations \\ to assess body composition using \\ anthropometric data in \\ Chilean preschool children
}

Background: Validated equations to measure body composition in Chilean preschool children are lacking, since the only international equation available was developed in obese, and undernourished caucasian children. Due to the increase in overweight and obesity in Chile, it is essential to have simple and reliable instruments to assess children. Aim: To design and validate anthropometric equations to estimate body fat (BF) and total body water (TBW) to be applicable in children aged 3 to 5 years, using deuterated water dilution as a reference method. Materials and Methods: Weight, height, circumferences and skinfold thickness were evaluated in 238 children aged 3 to 5 years, attending Junta Nacional de Jardines Infantiles (JUNI). Several equations were developed using multiple regression models to estimate body water and fat. The proposed equations were validated against a sample of 77 children previously evaluated, using Bland and Altman agreement analysis, that compares average differences of both methods with the average of predicted and estimated values. Results: New equations for TBW and BF were obtained, accounting for $86 \%$ and $72 \%$ in estimated variability. The agreement analysis showed a mean difference $0.001 \pm 0.32$ and $0.07 \pm 0.56 \mathrm{~kg}$, for TBW and BF, respectively, confirming the validity of the equations. Conclusions: The new validated equations in chilean children aged 3 to 5 years, have an adequate and predictive capacity, especially useful for evaluating TBW and BF change in cohorts (Rev Méd Chile 2008; 136: 433-41).

(Key words: Adipose tissue; Body composition; Body water; Child, preschool)
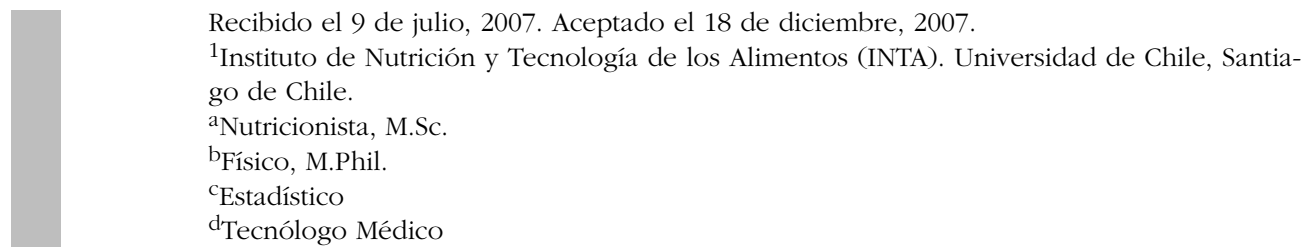

Correspondencia a: Marcia Velásquez. Instituto de Nutrición y Tecnología de los Alimentos (INTA), Laboratorio de Metabolismo Energético e Isótopos Estables, Universidad de Chile. El Líbano 5524, Teléfono: 9781462. Fax: 2931268. E mail: mvelasq@inta.cl - gsalazar@inta.cl 
$\mathrm{D}$ urante el proceso de crecimiento y desarrollo se producen una serie de cambios en la composición corporal (CC), principalmente en el almacenamiento y distribución del tejido muscular, óseo y adiposo, de acuerdo a edad y sexo. La niñez es un período de intensa actividad metabólica, con un desarrollo sostenido de la masa muscular y ósea hasta alcanzar la vida adulta. Las variaciones en estos componentes corporales dependen de la edad, sexo, grupo étnico, herencia, alimentación, actividad física, salud, ambiente físico y psicosocial ${ }^{1,2}$.

Los indicadores de carácter global más ampliamente usados son peso y talla, mas ellos no proporcionan información sobre la composición corporal. La medición de pliegues cutáneos, representa el promedio de la grasa subcutánea; la proyección de este valor a la cantidad de grasa total en el cuerpo, dependerá del número y tipo de pliegues y finalmente de la ecuación utilizada.

La influencia étnica sobre la composición corporal es clara. En asiáticos se ha encontrado mayor proporción de grasa corporal en relación al valor de índice de masa corporal (IMC) y grasa troncal ${ }^{3}$. Se ha demostrado que los niños hispánicos en Estados Unidos de Norteamérica (USA), tienen mayor grasa corporal desde que nacen, comparados con caucásicos y afroamericanos ${ }^{4,5}$.

Existen otras ecuaciones predictivas en un grupo más pequeño de preescolares chilenos, que no han sido validadas. Hasta el momento, la ecuación más utilizada en Chile es la de Brook ${ }^{6}$, que en promedio subestima la grasa corporal (GC) en niños preescolares chilenos (eutróficos, sobrepeso y obesos) en $5,9 \% 7,8$. Las ecuaciones de regresión para grasa corporal desarrolladas en una población específica, puede adolecer de fallas sistemáticas en su capacidad predictiva en niños:

a) Uso de algunas constantes propuestas para adultos, lo que influye sobre los resultados.

b) El tamaño de la muestra es insuficiente. Un ejemplo es la ecuación de Slaughter para el grupo de niñas, que evaluó sólo a 16 niñas entre 8 y 16 años?.

c) En el caso de la ecuación de Brook, el problema reside en el tipo de muestra utilizada (niños con talla baja y obesos), la cantidad insuficiente de niños (30 niños) para el amplio rango de edad (1 a 11 años) $)^{6}$.
En el presente trabajo se evaluaron más de 200 niños, utilizando las variables predictivas de peso, talla, pliegues cutáneos y circunferencias, junto a sexo, edad y etnia (tanto para agua como grasa corporal). Los valores predichos por las ecuaciones antropométricas fueron comparados contra los valores medidos por un método de referencia.

Entre los estándares de oro para evaluar composición corporal, la dilución isotópica mide la proporción más importante del cuerpo, agua corporal total (50\%-60\%). La grasa corporal total, evaluada indirectamente con dilución isotópica, tiene una excelente concordancia acuerdo en niños de 8-12 años, contra GC medida con el estándar de evaluación de composición corporal por cuatro compartimentos ${ }^{10,11}$. Adicionalmente, la técnica de dilución isotópica es inocua, no invasiva, sencilla, reproducible y puede ser utilizada directamente en trabajos de campo, en este caso en el jardín infantil.

El primer objetivo de esta investigación es estimar agua y grasa corporal total en niños de 3 a 5 años de edad; con estos datos se revisó el comportamiento de dos ecuaciones propuestas anteriormente, las cuales no han sido validadas en nuestra población. Si no se confirma la validez de estas ecuaciones, se diseñarán y validarán nuevas ecuaciones antropométricas, combinando mediciones de pliegues, edad, peso y talla, utilizando como método de referencia la dilución isotópica.

\section{MATERIAL Y MÉTODOS}

Sujetos. Se evaluaron niños preescolares de 3 a 5 años de edad, asistentes a los Jardines Infantiles de la Junta Nacional (JUNJI) de tres comunas de la ciudad de Santiago (La Florida, Nuñoa y Peñalolén). Entre los criterios de exclusión se consideró la desnutrición, la morbilidad, ingestión de medicamentos que alteraren la CC, edad mayor a 5 años.

Los niños fueron clasificados en eutróficos, sobrepeso y obesos, según el indicador peso para la talla (National Centre for Health Statistics, NCHS), propuesto por el Ministerio de Salud para los niños $<6$ años en Chile, hasta la fecha.

Para el diseño de ecuaciones, se estableció la muestra utilizando el criterio de Lohman ${ }^{12}$, que demostró que con un mínimo estadístico de 100 
niños se puede construir una ecuación antropométrica para GC, con un $\mathrm{R}^{2}=0,88$, con un límite de confianza inferior a 0,79 . Tomando en cuenta la variabilidad de estado nutricional (normal, exceso de peso), más la posible pérdida en la toma de muestra, se seleccionaron 238 niños. Para validar la ecuación obtenida con la muestra mencionada, se utilizaron los datos medidos anteriormente en un grupo similar de 77 niños (45 mujeres y 41 hombres). El consentimiento fue firmado por todos los padres o tutores de los participantes; dicho consentimiento había sido previamente aprobado por el Comité de Ética del Instituto de Nutrición y Tecnología de los Alimentos (INTA) de la Universidad de Chile.

Antropometría. La evaluación de cada variable antropométrica se repitió tres veces, obteniéndose el promedio, aplicando las técnicas de medición establecidas por Frisancho ${ }^{13}$ y Velásquez ${ }^{14}$. Para clasificar el estado nutricional mediante Peso/ Talla, se utilizó el puntaje Z, con referencia a las tablas NCHS, con los rangos sugeridos por la OMS y el Ministerio de Salud de Chile ${ }^{15}$. La antropometría braquial se realizó utilizando las fórmulas de Cachera-Rolland ${ }^{16}$.

Se midió peso corporal en una balanza portátil con plataforma (capacidad, $60 \mathrm{~kg}$ y $100 \mathrm{~g}$ de precisión marca EXCEL, modelo HW60-Santiago, Chile). La estatura se midió en el estadiómetro metálico de la misma balanza, con una escala de 1 a $200 \mathrm{~cm}$ y una precisión de $0,5 \mathrm{~cm}$. Se midieron los pliegues cutáneos (bíceps, tricipital, suprailíaco, subescapular y pantorrilla), utilizando un plicómetro marca Lange con una precisión de $1 \mathrm{~mm}$. La circunferencia de muñeca, brazo, cintura, cadera y pantorrilla, fueron medidas con una cinta métrica flexible con una escala de $10 \mathrm{~mm}$ (error $1 \mathrm{~mm}$ ).

Dilución isotópica. La medición de agua corporal total se hizo en los niños con ayuno de ocho horas. En algunos casos se autorizó la ingesta de leche o jugo (100-200 ml) hasta $2 \mathrm{~h}$ con anterioridad al examen, dada la pequeña edad de los niños. Se verificó que no hubiesen realizado actividad física por lo menos $12 \mathrm{~h}$ antes del examen y que tuviesen la vejiga vacía.

Para medir agua corporal total (ACT), se utilizó el método de dilución isotópica (protocolo de Plateau), el cual considera dos puntos de medición: una muestra basal previa a la ingestión del isótopo y otra muestra post dosis, al final del tiempo de equilibrio del isótopo en los fluidos corporales $(2,5 \mathrm{~h})^{17,18}$. Después de la recolección de la muestra basal, se administró una dosis de óxido de deuterio $\left(\mathrm{D}_{2} 0\right)$ de $1,5 \mathrm{~g} / \mathrm{kg}$ a $99 \%$ diluido a $50 \%$ y luego, $20 \mathrm{ml}$ de agua, para asegurar la total ingestión de la dosis. Durante el tiempo de equilibrio, los niños permanecieron en actividades recreativas de tipo sedentarias dentro de la misma sala. Se recolectó toda orina producida en ese período, para considerar las pérdidas eventuales de dosis. Las muestras de saliva fueron analizadas en triplicado, por espectrometría de masas en el Laboratorio de Metabolismo Energético e Isótopos Estables (INTA) de la Universidad de Chile.

A partir del cálculo de ACT, se calculó la masa libre de grasa total (kg), asumiendo los coeficientes de hidratación para niños de 3 a 5 años de edad, propuestos por Fomon ${ }^{19}$ y corregidos por Schoeller ${ }^{20}$. Se calculó la grasa corporal total, restando la masa libre de grasa del peso corporal.

Estadística. Se utilizó el Software Epi Info para evaluar el estado nutricional. En el software Statistic se evaluó la estadística descriptiva (análisis de medias, porcentajes, desviación estándar y valores mínimos y máximos). El test Levene permitió evaluar la homogeneidad de varianzas, tests paramétricos para las varianzas homogéneas y tests no paramétricos para aquellos casos en que las varianzas no cumplieron con la condición de homogeneidad ${ }^{21}$. En este último caso, se aplicaron los test Mann Whiney U y Kruskal-Wallis, para diferencias estadísticas. Además, se utilizó el test de correlación de Pearson, para establecer asociaciones entre variables ${ }^{21}$.

Se aplicaron los test para variables dependientes y test Shapiro-Wilkonson, para evaluar normalidad de las variables a ingresar en las ecuaciones antropométricas. Se empleó el programa SAS 6.0 en el diseño de las ecuaciones antropométricas.

Para la validación de las ecuaciones, se utilizó análisis de concordancia según Bland-Altman, que exige $95 \%$ de nivel de confianza $(-2 \mathrm{DE} \text { y }+2 \mathrm{DE})^{22}$. Este tipo de análisis permitió verificar si la medición por dos métodos es comparable, ya que una correlación adecuada no significa necesariamente que ambos métodos concuerden.

Además se realizó un análisis residual para evaluar la magnitud del acuerdo de los valores entregados por las ecuaciones antropométricas 
propuestas y el patrón de referencia (capacidad predictiva de las ecuaciones diseñadas $)^{22}$.

\section{Resultados}

La distribución de estado nutricional en esta población fue de $22 \%$ de obesos, $38 \%$ de sobrepeso y $40,3 \%$ de eutróficos según peso para la talla (NCHS). Se encontraron diferencias en el peso, talla, circunferencias y pliegues cutáneos, según estado nutricional ( $\mathrm{p}=0,0001)$.

Se encontraron diferencias significativas en todas las variables determinadas por el método de dilución isotópica con deuterio (Tabla 1). El porcentaje de ACT fue 60,3\% y 59\% en niños y niñas eutróficos, $58,1 \%$ y $56,5 \%$ en niños con sobrepeso y $56,7 \%$ y $55,0 \%$ para niños/as obesos, respectivamente. Con el incremento del peso, aumentó el porcentaje de grasa corporal; en niños y niñas eutróficos fue de 20,3\% y 23,7\%, versus 23,6\% y $26,2 \%$ en aquellos preescolares con sobrepeso y (26,5\% y 30,0\%) en obesos, respectivamente. a) Ecuaciones desarrolladas en estudios previos. Los resultados de la ecuación antropométrica de Salazar et al se compararon con los valores medidos por dilución isotópica para agua y grasa corporal ${ }^{7}$ (Tabla 2).

La ecuación de Salazar et al para ACT evaluó adecuadamente esa variable, con una diferencia promedio de 0,28 litros, con una $\mathrm{DE}=0,68$ litros; además existe una distribución homogénea de las diferencias residuales y una buena asociación entre los valores predichos y medidos $(r=0,89)$. La ecuación explica 79\% de la variabilidad en ACT.

En el caso de la GC, la diferencia promedio entre lo estimado y medido fue $0,68 \mathrm{~kg}$, pero no existe una distribución homogénea de los valores de GC estimados. Aunque existe una correlación de $r=0,78$ la ecuación tiende a sobreestimar la GC de niños magros y subestimar para los niños obesos.

b) Ecuaciones desarrolladas en el estudio actual. Por lo anteriormente descrito, la ecuación de Salazar et al, de grasa corporal no se puede utilizar, lo que obligó a construir nuevas ecuaciones para agua y grasa corporal, para la evaluación

Tabla 1. Estado nutricional de la población muestral según peso/talla por sexo ( $n=238$ )

\begin{tabular}{|lcccccc|}
\hline Clasificación & \multicolumn{2}{c}{ Hombres } & \multicolumn{2}{c}{ Mujeres } & \multicolumn{2}{c|}{ Total } \\
Z-Peso/Talla & $\mathrm{n}$ & $\%$ & $\mathrm{n}$ & $\%$ & $\mathrm{n}$ & $\%$ \\
\hline Eutrófico & 47 & 41,2 & 49 & 39,5 & 96 & 40,3 \\
Sobrepeso & 43 & 37,7 & 47 & 37,9 & 90 & 37,8 \\
Obeso & 24 & 21,1 & 28 & 22,6 & 52 & 21,8 \\
Total & 114 & 100 & 124 & 100 & 238 & 100 \\
\hline
\end{tabular}

Tabla 2. Ecuaciones antropométricas Salazar et al 20037

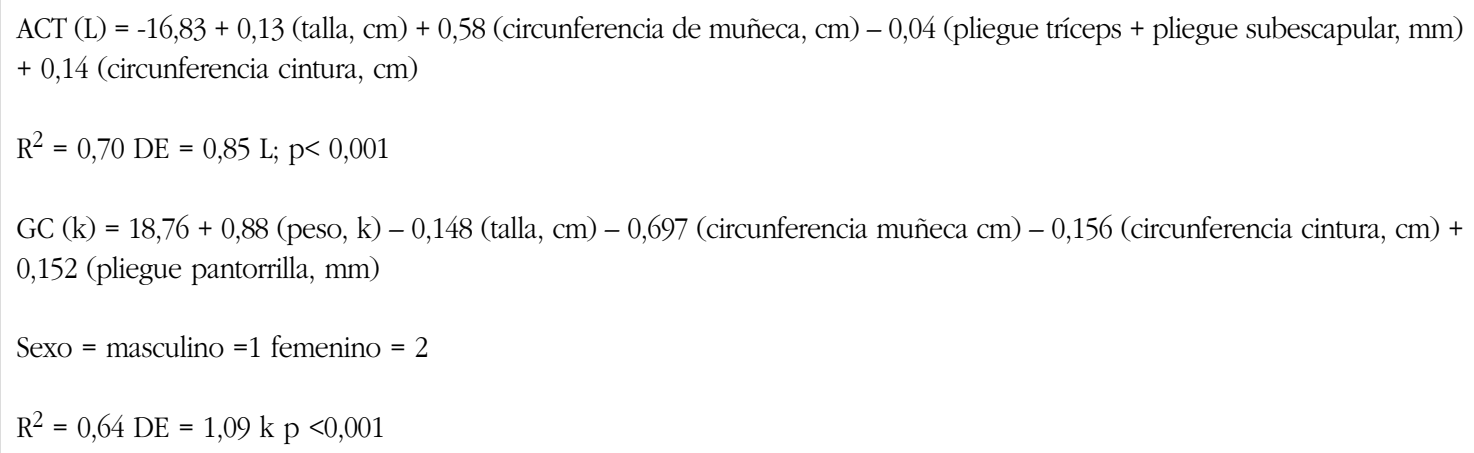


poblacional e individual. En la Tabla 3 se presentan las nuevas ecuaciones obtenidas, que poseen una correlación adecuada para agua $(\mathrm{r}=0,93)$ y grasa $(r=0,85)$.

c) Validación de nuevas ecuaciones propuestas. Para validar estas ecuaciones, se compararon los resultados de ACT y GC, con los valores medidos en una muestra independiente de 77 niños preescolares (3-5 años).
Los resultados muestran que en la ecuación para ACT, se obtuvo una diferencia promedio de 0,07 L ( $\mathrm{DE}=0,61 \mathrm{~L})$; el análisis residual evidencia una distribución homogénea. La asociación entre los valores obtenidos por la fórmula y el patrón de referencia, fue adecuada $(\mathrm{r}=0,89)$ (Figuras $1 \mathrm{a}, \mathrm{b})$, sin existencia de sesgo en las diferencias residuales.

En los valores estimados por la ecuación de GC, se obtuvo una diferencia promedio de $-0,07 \mathrm{~kg}$ y una DE $0,77 \mathrm{~kg}$, con una distribución homogénea

\section{Tabla 3. N uevos modelos antropométricos para determinar C $C$ en niños de 3-5 años}
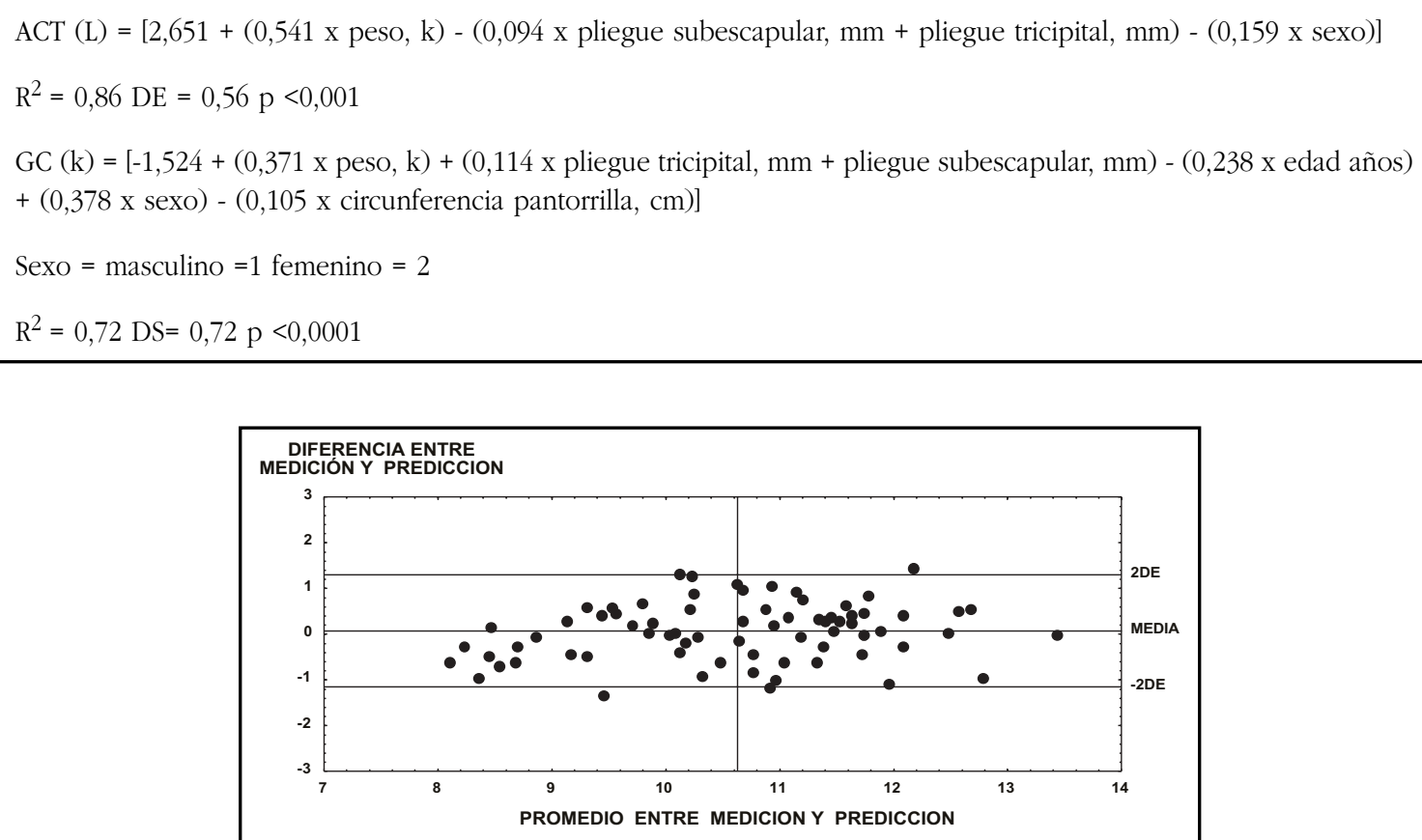

Figura 1a. Concordancia agua corporal total y ecuación antropométrica $(0,07)$. (Vélasquez et al) $(n=77)$.

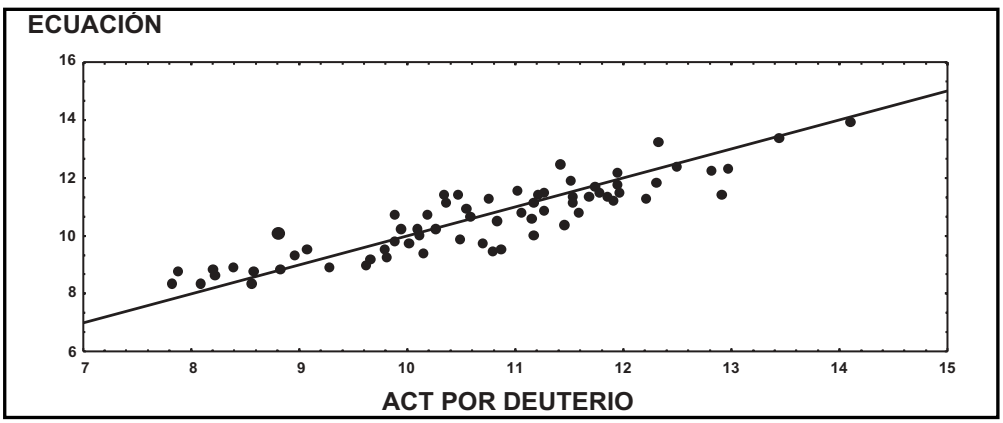

Figura 1b. Scatterplot a partir de deuterio y ecuación antropométrica para agua corporal $(r=0,89)$. (Velásquez et al) $(\mathrm{n}=77)$. 
en las diferencias residuales y una buena comparación entre el promedio del valor estimado y medido $(r=0,82)$ (Figuras $2 \mathrm{a}$ y $2 \mathrm{~b}$ ).

En conclusión, las nuevas ecuaciones tienen una mejor correlación entre las variables medidas y estimadas, una menor DE y una distribución homogénea en los puntos para la evaluación de ACT y GC.

\section{DisCUSIÓN}

Existe evidencia que la obesidad infantil ${ }^{23}$ está aumentando en nuestro país y en otros países de habla hispana ${ }^{24,25}$. La situación nutricional en Chile también ha cambiado rápidamente. Las cifras de obesidad en preescolares fue $9,7 \%$ en 2000 en el ámbito nacional y JUNJI reportó $10,8 \%$ en $2003^{26}$. La obesidad infantil ha pasado a constituir un problema nutricional importante en nuestro país, y por ello se ha realizado una investigación explorando el patrón de actividad física, hábitos alimentarios, necesidades de energía y CC en niños asistentes a jardines de la JUNJI ${ }^{27}$.

Por otro lado, la edad preescolar es clave para la generación de conductas adecuadas respecto de la alimentación y actividad física, con su resultante, un estado nutricional y crecimiento adecuado. La evaluación de la composición corporal en este grupo de edad resulta, entonces, un instrumento clave para ayudar en la prevención, control y seguimiento de la salud infantil. Existen pocos trabajos publicados en Chile con datos de composición corporal en niños, especialmente a temprana edad, a excepción del estudio en niños de 12 años promedio de edad ${ }^{28}$.

Adicionalmente, el componente de GC y su distribución es el principal factor de riesgo asocia-

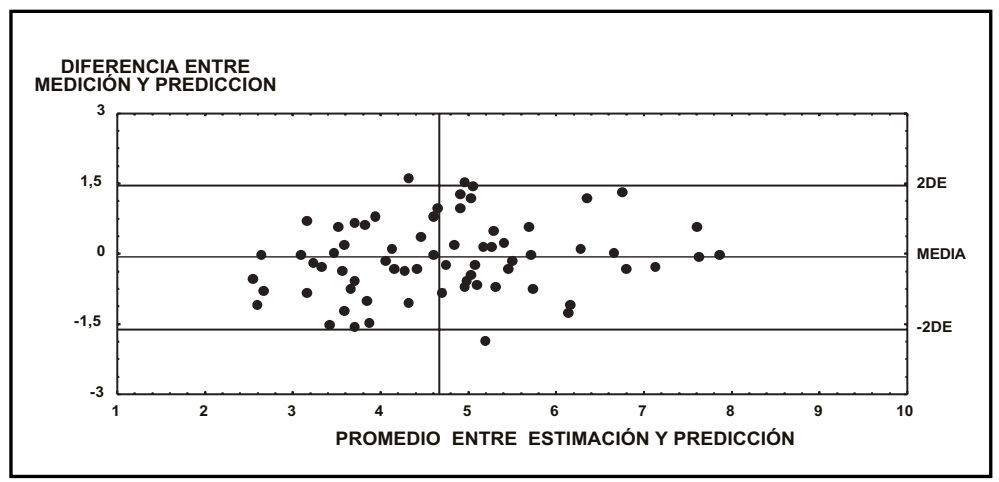

Figura 2a. Concordancia grasa corporal total y ecuación antropométrica $(-0,07)$. (Velásquez et al) $(n=77)$.

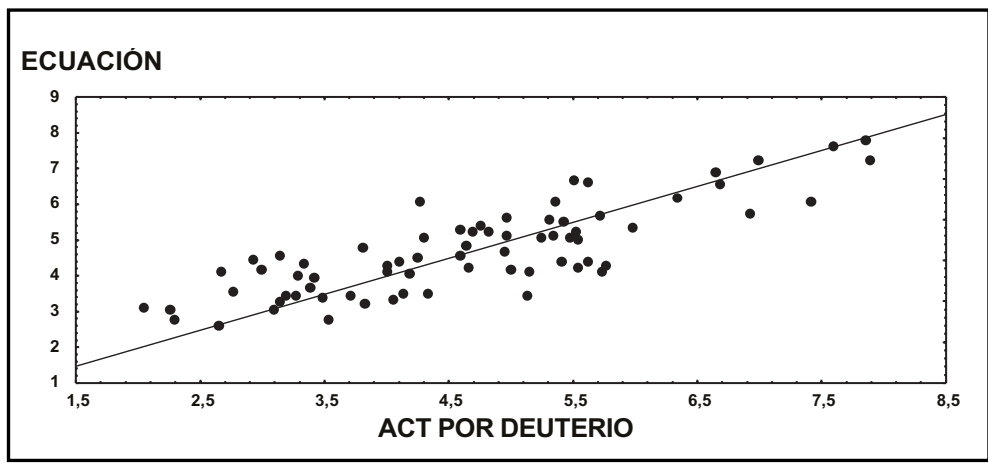

Figura 2b. Scatterplot a partir de deuterio y ecuación antropométrica para grasa corporal $(\mathrm{r}=82)$. 
do a morbilidad en la edad infantil ${ }^{29}$. Datos obtenidos en población hispana, indican que los niños de dicha ascendencia tienen mayor GC que la población caucásica desde que nacen ${ }^{30}$.

En este estudio se encontraron diferencias significativas en ACT según estado nutricional ( $\mathrm{p}$ $<0,0001)$. En comparación con los datos de Fomon ${ }^{19}$, АCT (\%) fue menor en niños eutróficos (60\% vs 64\%). El agua expresada en litros es mayor en varones que niñas, similar a lo mostrado por Ellis et $\mathrm{al}^{4}$ en niños hispanos, Bray et $\mathrm{al}^{30}$ en niños africanos de 10 años y Wells et al ${ }^{31}$ en niños británicos de 8 a 12 años. De este modo, la mayor cantidad de grasa corporal se reflejará en una disminución de la densidad corporal y el ACT.

En relación a GC (\%), existe una diferencia significativa por sexo y estado nutricional, siendo mayor en las niñas. Las niñas chilenas con estado nutricional normal tienen una cantidad de grasa similar a las hispanas $(23,7 \%)^{4}$ y mayor que lo establecido por Fomon $(17 \%)^{19}$, mientras que las niñas hispanas de la misma edad en USA tienen más grasa $(26 \%)^{32}$. En grasa en kilos hubo diferencia significativa sólo en eutróficos, siendo mayor en mujeres, $4,2 \mathrm{~kg}$ vs $4,4 \mathrm{~kg}$ en niñas hispanas. Otros autores también encontraron que las niñas hispanas tienen mayor GC, incluso desde el nacimiento 33,34 . Otros trabajos proponen que en niños pequeños es mejor utilizar la evaluación de grasa corporal en kilos, ya que utilizar el porcentaje de grasa produce resultados sesgados, debido a la variación conjunta de peso en los niños ${ }^{4,5}$.

Los resultados encontrados para grasa corporal (kg) no permitieron validar la ecuación para GC anteriormente propuesta por Salazar et $\mathrm{al}^{7}$. Se estima que la inclusión de un número menor de niños ( $n=117$ ) en la muestra utilizada para el diseño de la ecuación, podría explicar en parte este resultado.

Se procedió, por tanto, a construir una nueva ecuación para grasa corporal $(\mathrm{kg})$, en un grupo de 238 niños y niñas y determinar la validez de ellas, en un grupo independiente de 77 niños.

En la nueva ecuación para grasa (en kilos), se obtuvo una diferencia promedio de $1,5 \%$ respecto del patrón de referencia, sin diferencias significativas por análisis residual, ni sesgo de acuerdo a la cantidad de grasa corporal. Para la ecuación de GC, el porcentaje de la diferencia encontrada en la comparación de Bland y Altmann fue menor a 5\% planteado en esta investigación, y está en el rango hallado en otros modelos $(3 \%-9 \%)^{35}$. Además, no hubo diferencias, siendo independiente la cantidad de grasa corporal. Por tanto, los resultados permiten validar las nuevas ecuaciones propuestas.

Una fuente de error en una ecuación diseñada, aplicada en otros grupos etáreos diferentes del original, puede producir variaciones en el resultado, por factores étnicos, estructura corporal, entre otros. $\mathrm{Al}$ respecto, en niños ingleses, Wells et al ${ }^{11}$ determinaron que las ecuaciones antropométricas de Lohman, Weststrate-Deurenberg, Slaughter et al, Johnston et al, y Brook subestiman la GC, mientras que las ecuaciones para bioimpedancia eléctrica de Davies et al y Danford et al sobreestiman el porcentaje de grasa, con relación a un modelo de 4 compartimentos. Esto es importante, porque aunque son niños caucásicos en general, la evaluación en períodos, poblaciones o con diseños diferentes, influye en los resultados.

En Estados Unidos de Norteamérica, Goran et $\mathrm{al}^{34}$ destacaron que las ecuaciones de Slaughter et al y la de Kushner et al sobrestiman la grasa en niños, contra la medición de GC, hecha por DEXA en niños norteamericanos ${ }^{36}$. Reilly et al también compararon la ecuación de Brook et al en niños de 7 a 9 años de edad utilizando como patrón DEXA, señalando que esta ecuación proporcionaba errores de $19,8 \%$ en niñas $(r=0,21)^{37}$.

Por lo tanto, la ecuación diseñada para párvulos en el Reino Unido (Brook, 1972), subestima GC (\%) en los niños preescolares de nuestra muestra, especialmente en las niñas (6-8\%). Esta diferencia podría deberse al tamaño de la muestra, características de inclusión, estado nutricional, metodología y sobre todo a los coeficientes de hidratación utilizados $(0,73 \%)$ en las propuestas de Brook, Slaughter y Ellis ${ }^{38}$.

A medida que los niños aumentan en edad, tienen menor porcentaje de hidratación y esta proporción disminuye hasta llegar al valor de $0,73 \%$ en adultos. Una aplicación incorrecta de este coeficiente induce a la sobrestimación de grasa. Slaughter et al señalan que estimar la densidad corporal utilizando una constante válida para adultos, sobreestima la grasa en niños entre $3,9 \%$ y $4,5 \% 39$.

El otro factor a considerar, es que las metodologías utilizadas tienen una influencia sobre los modelos obtenidos. Lo más correcto es proponer 
modelos en base a la evaluación de cuatro compartimentos, pero esto suele ser más caro y requiere mayor personal y medios. En nuestro caso, al utilizar la medición de ACT por dilución isotópica, que evalúa el compartimiento corporal mayor (50\%-60\% del peso corporal) obtuvimos necesariamente un error menor, los que han sido demostrados por otros autores ${ }^{40}$.

Este trabajo ha mostrado que no es válido, en general, evaluar la CC mediante ecuaciones propuestas para grupos diferentes, con una realidad

\section{REFERENCIAS}

1. Restrepo MT. Evaluación del estado nutricional. En: Estado nutricional y crecimiento físico: Antioquia: Editorial Universidad; 2000; 100-26.

2. Van Loan M. Total body composition, birth to old age. In: Human Body Composition. Roche AF, Heymsfield SB, Lowman TG. Editors. Human Kinetics, Champaign (USA) 1996; 456-61.

3. Malina RM, Huang YC, Brown KH. Subcutaneous adipose tissue distribution in adolescent girls of four ethnic groups. Int J Obes Relat Metab Disord. 1995; 19: 793-7.

4. Ellis K, Abrams AS, Wang WW. Body composition of a young, multiethnic female population. Am J Clin Nutr 1997; 65: 724-31.

5. Ellis K, Abrams AS, Wang WW. Body composition of a young, multiethnic male population. Am J Clin Nutr 1997; 66: 1323-31.

6. Ввоок CGD. Determination of body composition of children from skinfolds measurements. Arch Dis Child 1971; 48: 182-4.

7. Salazar GR, Rocha Ma, Mardones F. ¿Es útil la antropometría para estimar la composición corporal en niños preescolares?. Rev Chil Pediatr 2003; 74: 37-45.

8. Velásquez RM. Diseño y validación de ecuación antropométricas para evaluar composición corporal en niños preescolares chilenos. Universidad de Chile INTA; 2003 Tesis de grado (MSc).

9. Slaughter mH, lohman TG, Bolleau Ra, Horsurll CA, Stilman RJ, Van Loan MD. Skinfolds equations for estimation of body fatness in children and youth. Hum Biol 1988; 60: 709-23.

10. Davis Ps, Wells JK. Calculation of total body water in infancy. Am J Clin Nutr 1994; 48: 490-5. étnica o epidemiológica distinta, siendo particularmente relevante estos aspectos en niños en crecimiento. Se puede concluir que las ecuaciones diseñadas tanto para agua como GC en el presente trabajo, demostraron ser confiables y tener una capacidad predictiva para ser utilizadas en la evaluación de CC en niños preescolares chilenos. Finalmente, se propone que las ecuaciones diseñadas y validadas puedan utilizarse en evaluación clínica y de salud pública, pues entregarán resultados más confiables a nivel grupal, e incluso individual.

11. Wells JC, Fuller NJ, Dewit O, Fewtrell MS, Elia M, Cole JJ. Four-component model of body composition in children: density and hydration of fatfree mass and comparison with simpler models. Am J Clin Nutr 1999; 69: 904-12.

12. lohman G, Caballero B, Himes JH, Hunsberger S, ReID R, Stewart D. Body composition assessment in American Indian children. Am J Clin Nutr 1999; 69: 764S-766S.

13. Frisancho R. New norms of upper limb fat and muscle areas for assessment of nutritional status. Am J Clin Nutr 1981; 34: 2540-5.

14. Velásquez RM. Métodos antropométricos para la evaluación del estado nutricional en adultos. La Paz. Ministerio de Desarrollo Humano, Secretaría Nacional de Salud, UPAN/OMS/OPS. 1995: 24-30.

15. BARRERA GM. Estándares antropométricos para evaluación del estado nutritivo. Santiago: Instituto de Nutrición y Tecnología de Alimentos (INTA) Universidad de Chile. 2004: 7-47.

16. Cachera-Rolland F, Branbilla P, Manzoni P, Akrout M, Siron S, Maschio A et al. Body composition assessment en the basis of arm circumference and triceps skinfold thickness: A new index validation by magnetic resonance imaging. Am J Clin Nutr 1997; 65: 1709-13.

17. Schoeller DA, Kushner RF, TaYlor P, Dietz WH, BANDINI L. Measurement of total body water: isotope dilution techniques. Report of the Sixth Ross Conference on Medical Research Ross Laboratories, Ohio. Am J Clin Nutr 1985: 24-9.

18. SAlAzAR G, ViO F. Deuterium equilibration time in infant's body water. Eur J Clin Nutr 1994; 48: 490-81.

19. Fomon S, Haschie F, Ziegler EE, Nelson SE. Body composition of reference children from birth to age 10 years. Am J Clin Nutr 1998; 35: 1169-75. 
20. Schoeller AD. Hydrometry. En Roche AF, Heymsfield SB, Lohman TG, eds. Human body composition. Human Kinetics, Champaign, 1996: 25-43.

21. Taucher E. Bioestadística. Santiago, Editorial Universitaria; 1997: 15-305.

22. Bland JM, Altman DG. Statistical methods for assessing agreement between two methods of clinical measurement. Lancet 1986; 1: 307-10.

23. Bautista-Castano I, Sangil-Monroy M, Serra-Majem L. Knowledge and gaps on the role of nutrition and physical activity on the onset of childhood obesity. Med Clin Barc 2004; 123: 782-93.

24. EluIs KJ. Body composition measurements. In: Kiess W, Marcus C, Wabitsch M. Editors: Obesity in Childhood and Adolescence Basal: New York Karger, 2004; 1-19

25. Pi-Sunyer F. Obesity a modern day epidemic. J Clin Endocrinol Metab 1999; 84: 3-7.

26. Albala, C, Vio F, Kain J. Obesidad: un desafío pendiente en Chile. Rev Méd Chile 1998; 126: 1001-9.

27. Vásquez F, Salazar G, Andrade M, Díaz E, Rojas J. Ingesta Alimentaria de preescolares obesos asistentes a los jardines infantiles de la JUNJI. Rev Chil Nutr 2004; 31: 100-8.

28. Barlett HL, Puhl SM, Hodgson JL, Buskirk ER. Fatfree mass in relation to stature: ratios of fat-free mass to height in children, adults, and elderly subjects. Am J Clin Nutr 1991; 53: 1112-6.

29. Burrows R. Prevención y tratamiento de la obesidad desde la niñez: la estrategia para disminuir las enfermedades crónicas no transmisibles del adulto. Rev Med Chil 2000; 128: 105-10.

30. Bray GA, Delany JP, Harsha DW, Volaufova, Champagne CC. Evaluation ob body fat in fatter and leaner $10 \mathrm{y}$ old African American and white: the Baton Rouge Children's Study. Am J Clin Nutr 2001; 73: 687-702.

31. Wells JC, Fuller NJ, Dewit O, Fewtrell MS, Elias M, Cole JJ. Four-component model of body compo- sition in children: density and hydration of fatfree mass and comparison with simpler models. Am J Clin Nutr 1999; 69: 904-12.

32. Hewitt JM, Going AD, Willians PD, Logman GT. Hydration of the fat-free body mass in children and adults. Implications for body composition assessment. Am J Physiol 1995; 265: 88-95.

33. Deurennerg P, Pieteres JL, Hautvast JGaJ. The assessment of the body fat percentage by skinfolds thickness measurements in childhood and young adolescence. Br J Nutr 1990; 63: 182-4.

34. Goran Mi, Driscoll P, Johnson R, Nagy TR, Hunter G. Cross-calibration of body-composition techniques against dual-energy-X-ray absorptiometry in young children. Am J Clin Nutr 1996; 63: 299-305.

35. Zemel B, Rielly E, Stallings V. Evaluation of methodology for nutritional assessment in children: Anthropometry, body composition and energy expenditure. Annu Rev Nutr 1997; 17: 211-35.

36. Kushner RF, Schoeller DA, Fjeld CR, Ford DL. Is the impedance index $(\mathrm{ht} 2 / \mathrm{R})$ significant in predicting total body water? Am J Clin Nutr 1999; 56: 835-9.

37. Reilly J, Willian J, Durnin J. Determination of body composition from skinfolds thickness: a validation study. Arch Dis Child 1995; 73: 305-10.

38. Butte N, Heinz C, Hopkinson J, Wong W, Shypailo R, Ellis K. Fat mass in infants and toddlers: Comparability of total body water, total body potassium, and body electrical conductivity, and dual-energy X-ray absorptiometry. J Pediatr Gastroenterol Nutr 1999; 29: 184-9.

39. Slaughter MH, Lohman RA, Boileau Ra, Horswill CA, Stillman RJ, VAN LOAN MD et al. Skinfold equation for estimation of body fatness in children and youth. Hum Biol 1986; 60: 709-23.

40. Kiess W, Marcus C, Wabitsch M. Obesity in Childhood and Adolescence. Pediatr Adolesc Med 2004; 9: 1-19. 